\title{
ON THE LOG-CONVEXITY OF TWO-PARAMETER HOMOGENEOUS FUNCTIONS
}

\author{
ZHEN-HANG YANG
}

Abstract. Suppose $f(x, y)$ is a positive homogeneous function defined on $\mathbb{U}\left(\subseteq \mathbb{R}_{+} \times \mathbb{R}_{+}\right)$, then call $\left(\frac{f\left(a^{p}, b^{p}\right)}{f\left(a^{q}, b^{q}\right)}\right)^{\frac{1}{p-q}}$ two-parameter homogeneous function and denote by $\mathcal{H}_{f}(a, b ; p, q)$. If $f(x, y)$ is third differentiable, then the log-convexity with respect to parameters $p$ and $q$ of $\mathcal{H}_{f}(p, q)$ depend on the sign of $J=(x-y)(x I)_{x}$, where $I=(\ln f)_{x y}$. As applications a group of chains of inequalities for homogeneous means are established, which generalize, strengthen and unify Tong-po Ling 's and Stolarsky's inequalities, and a reversed chain of inequalities for exponential mean (identic mean) is derived, which contains a reversed Stolarsky's inequality. Several estimations of lower and upper bounds of extended mean are presented.

Mathematics subject classification (2000): 26B25, 26D07, 26E60,26A48.

Key words and phrases: two-parameter homogeneous function, homogeneous mean, logarithmically convexity, chain of inequalities, estimation of lower and upper bounds.

\section{REFERENCES}

[1] B. C. CARLSOn, The logarithmic mean, Amer. Math. Monthly, 79, (1972), 615-618.

[2] P. CZINDER, Z. PÁLES, An extension of the Hermite-Hadmard inequality and an application for Gini and stolarsky means, Journal of Inequalities in Pure and Applied Mathematics, 5, (2) (2004) [ONLINE] Available online at http://jipam.vu.edu.au/images/167_03_JIPAM/167_03.pdf.

[3] C. GINI, Diuna formula comprensiva delle media, Metron, 13, (1938), 3-22.

[4] P. A. HÄsTÖ, A montonicity property of ratios of symmetric homogeneous means, Journal of Inequalities in Pure and Applied Mathematics, 3, (5) (2002) [ONLINE] Available online at http://jipamold.vu.edu.au/v3n5/013_02.pdf.

[5] GaO JiA, Jinde CAO, A new upper bound of the logarithmic mean, Journal of Inequalities in Pure and Applied Mathematics, 4, (4) (2003) [ONLINE] Available online at http://jipam.vu.edu.au/images/088_03/088_03_www.pdf.

[6] E. B. LeAch, M. C. Sholander, Extended mean values, Amer. Math. Monthly, 85, (1978), 84-90.

[7] E. B. LeACH, M. C. Sholander, Extended mean values II, J. Math. Anal. Appl., 92, (1983), 207-223.

[8] T.-P. Lin, The power mean and the logarithmic mean, Amer. Math. Monthly, 81, (1974), 879-883.

[9] Z. PÁLES, Inequalities for differences of powers, J. Math. Anal. Appl., 131, (1988), 271-281.

[10] F. QI, Logarithmically convexities of the Extended Mean values, Proc. Am. Math. Soc. 130, (6) (2002), 1787-1796.

[11] F. QI, Generalized weighted mean values with two parameters, Proceedings of the Royal Society of London, Series, Mathematical, Physical and Engineering Sciences, 454, (1978) (1998), 2723-2732.

[12] F. QI, On a two-parameter family of nonhomogeneous mean values, Tamkang Journal of Mathematics, 29, (2) (1998), 155-163.

[13] F. QI, Generalized Abstracted Mean Values, Journal of Inequalities in Pure and Applied Mathematics, 1, (1) (1999) [ONLINE] Available online at http://jipam-old.vu.edu.au/v1n1/013_99.pdf.

[14] K. B. StolarsKY, Generalizations of the Logarithmic Mean, Math. Mag., 48, (1975), 87-92.

[15] K. B. StOLARSKY, The power and generalized Logarithmic Means, Amer. Math. Monthly., 87, (1980), 545-548. 
[16] MING-BAo SUN, Inequality for two-parameter mean of convex function, Mathematics in Practice and Theory, 27, (3) (1997), 193-197.

[17] ZHEN-HANG YANG, Exponential mean and logarithmic Mean, Mathematics in Practice and Theory, 4, (1987), 76-78.

[18] ZHEN-HANG YANG, Inequalities for power mean of convex function, Mathematics in Practice and Theory, 20, (1) (1990), 93-96.

[19] ZHEN-HANG YANG, Inequalities for general mean of convex function, Mathematics in Practice and Theory, 33, (8) (2003), 136-141.

[20] ZHEN-HANG YANG, Simple discriminance for convexity of homogeneous functions and applications, Study in College Mathematics, 7, (4) (2004), 14-19. 